\title{
Effective Connectivity Within the Default Mode Network in Left Temporal Lobe Epilepsy: Findings from the Epilepsy Connectome Project
}

\author{
Cole J. Cook, ${ }^{1}$ Gyujoon Hwang, ${ }^{1}$ Jedidiah Mathis, ${ }^{2}$ Veena A. Nair, ${ }^{3}$ Lisa L. Conant, ${ }^{4}$ Linda Allen, ${ }^{5}$ \\ Dace N. Almane, ${ }^{6}$ Rasmus Birn, ${ }^{1,7}$ Edgar A. DeYoe, ${ }^{2,8}$ Elizabeth Felton, ${ }^{6}$ Courtney Forseth, ${ }^{6}$ \\ Colin J. Humphries, ${ }^{4}$ Peter Kraegel, ${ }^{4}$ Andrew Nencka, ${ }^{8}$ Onyekachi Nwoke, ${ }^{9}$ Manoj Raghavan, ${ }^{4}$ \\ Charlene Rivera-Bonet, ${ }^{10}$ Megan Rozman, ${ }^{4}$ Neelima Tellapragada, ${ }^{3}$ Candida Ustine, ${ }^{6}$ \\ B. Douglas Ward, ${ }^{8}$ Aaron Struck, ${ }^{5}$ Rama Maganti, ${ }^{5}$ Bruce Hermann, ${ }^{6}$ Vivek Prabhakaran, ${ }^{3,6,10}$ \\ Jeffrey R. Binder, ${ }^{4,7}$ and Mary E. Meyerand ${ }^{1,3,11}$
}

\begin{abstract}
The Epilepsy Connectome Project examines the differences in connectomes between temporal lobe epilepsy (TLE) patients and healthy controls. Using these data, the effective connectivity of the default mode network (DMN) in patients with left TLE compared with healthy controls was investigated using spectral dynamic causal modeling (spDCM) of resting-state functional magnetic resonance imaging data. Group comparisons were made using two parametric empirical Bayes (PEB) models. The first level of each PEB model consisted of each participant's spDCM. Two different second-level models were constructed: the first comparing effective connectivity of the groups directly and the second using the Rey Auditory Verbal Learning Test (RAVLT) delayed free recall index as a covariate at the second level to assess effective connectivity controlling for the poor memory performance of left TLE patients. After an automated search over the nested parameter space and thresholding parameters at $95 \%$ posterior probability, both models revealed numerous connections in the DMN, which lead to inhibition of the left hippocampal formation. Left hippocampal formation inhibition may be an inherent result of the left temporal epileptogenic focus as memory differences were controlled for in one model and the same connections remained. An excitatory connection from the posterior cingulate cortex to the medial prefrontal cortex was found to be concomitant with left hippocampal formation inhibition in TLE patients when including RAVLT delayed free recall at the second level.
\end{abstract}

Keywords: default mode network; dynamic causal modeling; temporal lobe epilepsy

\section{Introduction}

$\mathbf{E}$ PILEPSY IS THE fourth most common neurological disorder affecting 65 million people worldwide (Sirven and Shafer, 2014), and temporal lobe epilepsy (TLE) is the most common focal epilepsy (Fisher, 2013). The defining feature of epilepsy is a disposition to recurrent seizures, but the morbid- ity associated with epilepsy is not limited to the ictal and periictal states. Even in the interictal state, epilepsy is associated with a number of neurobehavioral and cognitive consequences (Helmstaedter and Witt, 2012; Lin et al., 2012). Especially prominent in TLE is impaired anterograde memory (Saling, 2009). The mechanisms underlying anterograde memory impairment are unclear. We hypothesize that disruption of

\footnotetext{
${ }^{1}$ Department of Medical Physics, University of Wisconsin-Madison, Madison, Wisconsin.

${ }^{2}$ Department of Radiology, Froedtert and Medical College of Wisconsin, Milwaukee, Wisconsin.

${ }^{3}$ Department of Radiology, School of Medicine and Public Health, University of Wisconsin-Madison, Madison, Wisconsin.

${ }^{4}$ Department of Neurology, Medical College of Wisconsin, Milwaukee, Wisconsin.

${ }^{5}$ Department of Neurology, Froedtert Hospital, Milwaukee, Wisconsin.

Departments of ${ }^{6}$ Neurology and ${ }^{7}$ Psychiatry, University of Wisconsin-Madison, Madison, Wisconsin.

${ }_{0}^{8}$ Department of Biophysics, Medical College of Wisconsin, Milwaukee, Wisconsin.

${ }^{9}$ School of Pharmacy, University of Wisconsin-Madison, Madison, Wisconsin.

${ }^{10}$ Neuroscience Training Program, University of Wisconsin-Madison, Madison, Wisconsin.

${ }^{11}$ Department of Biomedical Engineering, University of Wisconsin-Madison, Madison, Wisconsin.
} 
cerebral connectivity, specifically in the default mode network (DMN), may play an explanatory role.

The DMN differs between TLE patients and controls in terms of functional connectivity (e.g., McCormick et al., 2013; Zhang et al., 2010) and is implicated in various types of memory, including episodic (Buckner et al., 2008; Sestieri et al., 2011) and semantic memory (Binder et al., 2009). For example, in one study, verbal memory performance was correlated with functional connectivity of the posterior cingulate cortex (PCC) and bilateral hippocampi (regions of the DMN) in controls, while only functional connectivity of PCC and the left hippocampus correlated with verbal memory in left TLE (McCormick et al., 2013). DMN functional connectivity differences between TLE patients and controls are thought to be structural in origin (Voets et al., 2012; for reviews of connectivity differences in epilepsy, see Cataldi et al., 2013; Yaffe et al., 2015).

Effective connectivity, unlike functional connectivity that is often measured using temporal correlation, investigates the impact of one neural system over another (Friston, 2011b). Few studies have examined effective connectivity in the DMN in epilepsy patients and the authors know of none that have controlled for cognitive measures as a covariate in effective connectivity analysis. Prior investigations on effective connectivity of the DMN in epilepsy have used Granger causality (Luo et al., 2016; Wei et al., 2016). Granger causality is a model of effective connectivity on the blood oxygenation level-dependent (BOLD) signal. However, estimation of effective connectivity at the level of the BOLD signal may lead to incorrect directionality due to nonlinear hemodynamic response differences and Granger causality is not robust to the observation noise often exhibited in neuroimaging data (David et al., 2008; Friston, 2011a; Friston et al., 2013, 2014). Dynamic causal modeling (DCM; Friston et al., 2003) overcomes these shortcomings by modeling effective connectivity at the hidden (unobserved) neuronal level and accounts for hemodynamic differences using local hemodynamic parameters.

The objectives of this study are threefold. First, to compare verbal anterograde memory performance in TLE patients compared with controls. The hypothesis being that verbal memory would be impaired in the left TLE patients. Second, to investigate alterations in effective connectivity within the DMN between TLE patients and healthy controls. The hypothesis being that aberrant hippocampal connectivity would be found in TLE patients. Third, to determine the impact of controlling for verbal memory when comparing patterns of effective connectivity in TLE patients versus controls. In this analysis, verbal memory was included in the second level to determine its impact on group differences on effective connectivity in the DMN using spectral DCM (spDCM; Friston et al., 2014). If controlling for verbal memory impairment resolves the group differences in DMN effective connectivity, this would suggest that variance in verbal memory in and of itself can encapsulate most variation in DMN effective connectivity. If, however, differences in effective connectivity do not attenuate, then the effects of DMN disruption are potentially manifold with cognitive and neurobehavioral consequences varying between individuals. In this case, perhaps the effects of DMN disruption on other cognitive domains need to be examined and the role of modifying factors such as education and IQ needs to be explored before the clinical implications of DMN disruption in TLE can be fully appreciated.

\section{Methods}

\section{Participants}

Study inclusion criteria for TLE patients consisted of a diagnosis of TLE supported by two or more of the following: (1) described or observed clinical semiology consistent with seizures of temporal lobe origin, (2) electroencephalogram (EEG) evidence of either temporal intermittent rhythmic delta activity or temporal epileptiform discharges, (3) temporal onset of seizures captured on EEG, and (4) magnetic resonance imaging (MRI) evidence of mesial temporal sclerosis or hippocampal atrophy. Exclusion criteria for TLE patients included the following: (1) age at recruitment outside the range of 18-60 years, (2) estimated full-scale IQ <70, (3) inability to provide informed consent, (4) reported or directly observed semiological features that suggest extratemporal seizure origin, (5) presence of any lesions other than mesial temporal sclerosis and nonspecific white matter abnormalities on 3 T MRI with a dedicated epilepsy protocol, (6) an active infectious etiology of seizures, (7) suspected or confirmed evidence of active autoimmune or inflammatory process in the central nervous system, (8) contraindications for MRI, or (9) non-English speakers. Exclusion criteria for healthy controls included the following: (1) age outside of 18-60, (2) primary language other than English, (3) history of any learning disability, (4) history of brain injury or illness, substance abuse, or major psychiatric illness, (5) visual deficit not correctable by lenses, (6) active cardiac disease, (7) current use of psychoactive or vasoactive medications, (8) claustrophobia, anxiety, or cardiopulmonary disease, and (9) contraindications to MRI.

Inclusion for this investigation was restricted to patients identified with TLE lateralized to the left hemisphere (based on clinical and EEG data). The number of TLE patients who underwent video-EEG monitoring of spontaneous seizures, with unilateral left temporal epileptiform discharges and with mesial temporal sclerosis or hippocampal atrophy on MRI, is included in Table 1. Previous studies have demonstrated that unilateral temporal epileptiform discharges and mesial temporal sclerosis or hippocampal atrophy are highly correlated with the epileptogenic region (Holmes et al., 1996; Vollmar et al., 2018; Williamson et al., 1993). All participants were 30 years of age or older and had both MRI data and neuropsychological test results.

The initial study sample included 24 left TLE patients and 17 controls. Two patients were removed from the study due to excessive motion during imaging. The five oldest patients were removed from the sample to match the groups on mean age. In the final sample, 34 participants were included (Table 1); 17 left TLE patients (mean age $=41.04$ years, seven males) and 17 controls (mean age $=38.07$ years, eight males) from the two sites in the Epilepsy Connectome Project (ECP): the Medical College of Wisconsin (MCW) and the University of Wisconsin-Madison (UW). All participants but one TLE patient and one control were righthanded. All participants provided informed consent in accordance with the procedures of the MCW Institutional Review Board and were enrolled in the ECP, a National Institutes of Health-sponsored project jointly managed by MCW and UW. 
Table 1. Group Demographics Including Sample Size, Gender Ratio, Mean Ages, Education, Handedness, Mean DVARS, Mean RAVLT Scores, and Number of AEDs

\begin{tabular}{|c|c|c|c|}
\hline & Healthy controls & TLE patients & $\mathrm{p}$-Value \\
\hline Sample size & 17 & 17 & NA \\
\hline Gender (M/F) & $8 / 9$ & $7 / 10$ & 1 \\
\hline Age (years) & $38.07 \pm 7.28$ & $41.04 \pm 5.44$ & 0.189 \\
\hline Education (years) & $15.65 \pm 2.00$ & $15.88 \pm 2.15$ & 0.743 \\
\hline Handedness (R/L) & $16 / 1$ & $16 / 1$ & NA \\
\hline Mean DVARS & $44.52 \pm 4.98$ & $45.07 \pm 5.04$ & 0.751 \\
\hline RAVLT delayed free recall & $102.06 \pm 13.99$ & $86.88 \pm 18.47$ & 0.008 \\
\hline Spontaneous video-EEG monitoring & NA & 12 & NA \\
\hline Ictal epileptiform activity & NA & 11 & NA \\
\hline Mesial temporal sclerosis or hippocampal atrophy & NA & 6 & NA \\
\hline Number of AEDs & NA & $2.06 \pm 1.20$ & NA \\
\hline 0 & & 1 & \\
\hline 1 & & 5 & \\
\hline 2 & & 6 & \\
\hline 3 & & 2 & \\
\hline 4 & & 3 & \\
\hline Achenbach ASR & $55.88 \pm 6.07$ & $61.94 \pm 11.40$ & 0.10 \\
\hline Depression $(>70)$ & (1) & (4) & \\
\hline
\end{tabular}

Mean ages, mean education, and mean DVARS averaged over phase encode were compared using an independent two-sample t-test and gender ratios were compared using Fisher's exact test. RAVLT and Achenbach ASR scores were compared using Wilcoxon rank sum tests. pValues are given in the last row, where applicable.

TLE, temporal lobe epilepsy; M, male; F, female; R, right; L, left; DVARS, root-mean-square spatial variance per voxel of successive difference images; RAVLT, Rey Auditory Verbal Learning Test; EEG, electroencephalogram; AED, antiepileptic drug; ASR, adult selfreport; NA, not applicable.

TLE patients were primarily recruited from populations served by the Comprehensive Epilepsy Programs at MCW and UW, with some by referrals from community neurology practices or through self-referral after seeing a pamphlet or website posting. Healthy controls were recruited through advertisements. All participants were compensated for their time.

\section{MRI scanning}

MRI images were acquired on a 3 T GE MRI scanner (GE Healthcare Discovery MR750; Waukesha, WI) using a Nova 32-channel head coil. T1-weighted structural images were acquired using a three-dimensional (3D) gradientecho pulse (MPRAGE) sequence (repetition time $[\mathrm{TR}]=$ $604 \mathrm{msec}$, echo time $(\mathrm{TE})=2.516 \mathrm{msec}$, inversion time $=$ $1060 \mathrm{msec}$, flip angle $=8^{\circ}$, field of view $(\mathrm{FOV})=25.6 \mathrm{~cm}$, $0.8 \mathrm{~mm}$ isotropic). T2-weighted structural images were acquired using a 3D fast spin-echo (CUBE) sequence $\left(\mathrm{TR}=2500 \mathrm{msc}, \mathrm{TE}=94.398 \mathrm{msec}\right.$, flip angle $=90^{\circ}, \mathrm{FOV}=$ $25.6 \mathrm{~cm}, 0.8 \mathrm{~mm}$ isotropic).

Resting-state functional MRI (rsfMRI) data were acquired using an echo planar imaging sequence $(\mathrm{TR}=802 \mathrm{msec}$, $\mathrm{TE}=33.5 \mathrm{msec}, \mathrm{FOV}=20.8 \mathrm{~cm}$, flip angle $=50^{\circ}, 72$ slices, $2 \mathrm{~mm}$ isotropic voxels, multiband acceleration factor of 8) following structural imaging. Two sessions each containing a set of four 5-min resting scans were collected, as two pairs with opposite phase encoding totaling eight scans. For this study, data from just two resting-state runs per participant (total of $10 \mathrm{~min}$ ) were analyzed to have complete data from all participants; the first was acquired using an anterior/posterior phase encode and the second using the opposite. Participants were told to gaze at a white cross on a black background. In total, 722 volumes (361 per run) were used in this analysis.

\section{Behavioral measures}

All participants were administered the Achenbach Adult Self-Report (ASR; Achenbach and Newhouse, 2004). The Diagnostic and Statistical Manual of Mental Disorders (DSM) - oriented depressive problems score on the Achenbach ASR was of interest as depression has a high incidence rate in the TLE population (Theodore et al., 2012) and may impact memory performance. The mean T-score for depressive problems is given in Table 1.

Verbal learning and memory were assessed using the Rey Auditory Verbal Learning Test (RAVLT; Geffen et al., 1990; Rey, 1941; Schmidt, 2017). The RAVLT is a multitrial, word list learning task frequently used in clinical and research settings to assess verbal learning and memory. The RAVLT is among the most sensitive measures of anterograde memory pathology in left TLE patients (Grammaldo et al., 2006; Loring et al., 2008). In this task, 15 unrelated words were read aloud to participants, who were asked to freely recall as many words as possible. These same words were presented across five learning trials and the number of recalled words in each trial was summed to create a total learning score. A new series of 15 words was read to participants after the fifth trial and they freely recalled as many words as possible from the new list. Recall of the original list was then performed (short delay free recall). Finally, participants were asked to recall as many of the original 15 words as possible after a 30-min delay (long delay free recall). Age- and gender-corrected standard scores for the long delay free recall were used in the analysis (Table 1). 


\section{Data preprocessing and analysis}

rsfMRI data were initially slice time corrected using Statistical Parametric Mapping 12 (SPM12) version 6909 (The FIL Methods Group, 2016). Data preprocessing then followed the Human Connectome Project (HCP) minimal preprocessing pipeline version 3.4.0 (Glasser et al., 2013), which is primarily based on FMRIB Software Library (FSL; Jenkinson et al., 2012) and FreeSurfer (Dale et al., 1999; Fischl and Dale, 2000; Fischl et al., 2002). Processing of the structural T1-weighted and T2-weighted images included correction for magnetic inhomogeneity, alignment and registration to Montreal Neurological Institute (MNI) space and FreeSurfer's surface atlas, and volume segmentation. The rsfMRI data were corrected for spatial distortion, motion corrected, aligned to structural data, bias field corrected, normalized to a global mean, and masked using the final brain mask.

\section{Motion}

Image quality of each subject's run was checked using the root-mean-square spatial variance per voxel of successive difference image (DVARS) metric. DVARS measures the change in brain image intensity compared with the previous time point. It is calculated using the formula (Power et al., 2012),

$$
\operatorname{DVARS}_{i}=\sqrt{\left\langle\left[\Delta I_{i}(\vec{x})\right]^{2}\right\rangle}=\sqrt{\left\langle\left[I_{i}(\vec{x})-I_{i-1}(\vec{x})\right]^{2}\right\rangle}
$$

where $I_{i}(\vec{x})$ is the image intensity at location $\vec{x}$ on frame $i$. Mean DVARS is simply the average of DVARS across all frames.

\section{General linear model and region of interest extraction}

Data analysis was performed using SPM12 following the HCP preprocessing steps. The rsfMRI data were smoothed using a $6 \mathrm{~mm}$ Gaussian kernel. White matter and cerebrospinal fluid (CSF) segments were created in SPM12 from the T1-weighted image. Average white matter and CSF signals were obtained from rsfMRI data using their respective T1 segmentation masks. These average signals, six motion parameters, and motion parameter derivatives were then regressed out of the rsfMRI signal to minimize potential non-neural noise sources. Motion regressors were derived using FSL's MCFLIRT during HCP-style processing. Regression was performed using SPM12 with the FAST option for determining correlation structure.

Time courses from the six DMN regions in Van Dijk and colleagues (2010) were extracted following regression of nuisance variables using SPM12: PCC, medial prefrontal cortex (mPFC), bilateral lateral parietal cortices (left lateral parietal cortex [LLPC] and right lateral parietal cortex [RLPC]), and bilateral hippocampal formations (left hippocampal formation [LHF] and right hippocampal formation [RHF]). Table 2 lists MNI coordinates of the centers of the 6-mm-radius spherical seed regions (Van Dijk et al., 2010).

\section{$s p D C M$}

DMN seed regions were used in a fully connected spDCM (Friston et al., 2014). spDCM assumes a linear random differential equation of the following form:
Table 2. Montreal Neurological Institute Space Coordinates of the Default Mode Network Seed Regions

\begin{tabular}{lrcr}
\hline & \multicolumn{3}{c}{ MNI coordinates } \\
\cline { 2 - 4 } Region & \multicolumn{1}{c}{$X$} & $Y$ & $Z$ \\
\hline PCC & 0 & -53 & 26 \\
mPFC & 0 & 52 & -6 \\
LLPC & -48 & -62 & 36 \\
RLPC & 46 & -62 & 32 \\
LHF & -24 & -22 & -20 \\
RHF & 24 & -20 & -22 \\
\hline
\end{tabular}

These coordinates are those used in Van Dijk et al. (2010).

MNI, Montreal Neurological Institute; PCC, posterior cingulate cortex; mPFC, medial prefrontal cortex; LLPC, left lateral parietal cortex; RLPC, right lateral parietal cortex; LHF, left hippocampal formation; RHF, right hippocampal formation.

$$
\dot{x}(t)=\boldsymbol{A} x(t)+v(t)
$$

where $x(t)=\left[x_{1}(t), \ldots, x_{n}(t)\right]^{T}$ is a column vector corresponding to the hidden (unobserved) neuronal states of the $n$ regions of interest, $\dot{x}(t)$ is a length $n$ column vector of neuronal state time derivatives, $\boldsymbol{A}$ is an $n \times n$ matrix encoding effective connectivity, and $v(t)$ is an $n$-vector of endogenous fluctuations. The columns of the $\boldsymbol{A}$ matrix denote the region of neuronal activity origin and the rows denote the region being influenced. The estimated self-connection values, diagonal elements of the $\boldsymbol{A}$ matrix, in the spDCM output have units of $\mathrm{Hz}$ after exponentiation and multiplication by -0.5 (Park et al., 2017). Positive estimated self-connections indicate greater self-inhibition, while negative estimated self-connections represent less self-inhibition with respect to the prior (Park et al., 2017). Extrinsic connections, offdiagonal elements of the $\boldsymbol{A}$ matrix, have units of $\mathrm{Hz}$ and are excitatory when positive and inhibitory when negative. The cross-spectral density of the endogenous fluctuations and MRI observation noise are modeled as a power law

$$
g(\omega, \theta)=\alpha \omega^{-\beta}
$$

where $g$ is the cross-spectral density, $\omega$ is the frequency, $\theta$ is a collection of parameters used in modeling, and $\alpha$ and $\beta$ denote the amplitude and exponential power of the crossspectral density, respectively. The hemodynamic model described in Stephan and colleagues (2007) maps the hidden neuronal states to hemodynamic states. This model assumes that hemodynamic states are a function only of each region's neuronal state.

spDCM uses a Bayesian framework for estimation. Thus, priors are placed on all parameters previously discussed and the likelihood of observed data given these parameters can be calculated. Together, the priors and likelihood determine the posterior probability of the generative model's parameters using variational Laplace (Friston et al., 2007). See Friston and associates (2003) for empirical hemodynamic parameter priors and Friston and associates (2014) for neuronal model priors.

A potential relationship between each connection in the fully connected spDCM and the number of antiepileptic 
drugs (AEDs) used by patients was investigated in two ways: via correlation and a Wilcoxon rank sum comparing polytherapy versus monotherapy. Multiple comparisons were corrected for using false discovery rate.

\section{Parametric empirical Bayes modeling}

A parametric empirical Bayes (PEB) model was used to compare connection strengths and the most likely network model between TLE patients and controls. The PEB model used in this study is a hierarchical linear model in which the first level consists of estimated fully connected DCMs and the second level is a Bayesian linear regression model (Friston et al., 2016). The first level DCMs were the fully connected spDCMs estimated from each subject's data, which served as priors to constrain the variables in the Bayesian linear regression model. This allowed the variability in an individual subject's connection strengths to influence the second-level analysis, providing a benefit over other methods such as analysis of variance.

Two PEB models were investigated: one with and the other without RAVLT delayed recall as a covariate in the second level. The second-level Bayesian linear model for the first PEB model included the intercept and two variables: group (patient vs. control) and phase encode direction. The second PEB model included the same variables in the second-level Bayesian linear model as in the first, as well as standardized RAVLT delayed recall score. The RAVLT score was added as a covariate to control for interparticipant variation in memory abilities. A 1,-1 binary encode was used for both the group and phase encode variables in which control and AP phase encode were denoted with a 1 and patients and PA phase encode were denoted with a -1 . RAVLT scores were mean-centered.

\section{Bayesian model reduction and averaging}

After fitting both full PEB models, Bayesian model reduction (BMR) was used to reduce the number of parameters in each PEB model. BMR computes the posterior probability for the set of parameters and the model evidence for any nested model within the full model (Friston et al., 2016). These are used to calculate an approximation to the free energy, $F$, which is calculated as follows:

$$
F(\tilde{p}, \tilde{q})=E_{q}[\ln p(y \mid \theta)]-D_{\{K l\}}[q(\theta \mid \tilde{q}) \mid p(\theta \mid \tilde{p})]
$$

where $\tilde{p}$ denotes the sufficient statistics for the prior, $\tilde{q}$ denotes the posterior sufficient statistics, $E_{q}$ is the expectation with respect to the assumed posterior distribution $q, p$ is the distribution of the prior, $y$ is the data, $D_{\{K l\}}$ is the Kullback-Leibler divergence, and $\theta$ is the collection of parameters. The model with the highest free energy was chosen as the winning model.

Bayesian model averaging (BMA) was performed for the two PEB models after obtaining the probability for all possible PEB models (nested and full) separately via BMR. BMA averages over the PEB models, weighted by their model evidence, rather than choosing one final model. The final BMA PEB model parameters were then thresholded, keeping only those with strong evidence (posterior probability $>0.95$ )

\section{Results}

All statistical tests, excluding DCM and PEB models, were performed in R (R Core Team, 2017).

\section{Group demographics}

No significant group differences were found for age $\left(t_{29}=-1.345, p=0.189\right)$ or education $\left(t_{31}=-0.331, p=0.743\right)$ using an independent two-sample $t$-test assuming unequal variance. No significant gender differences were observed between groups as evaluated with Fisher's exact test $(p>0.99)$.

\section{Behavioral measures}

No significant differences in Achenbach ASR scores for depressive problems on DSM-oriented scales were found between groups using a Wilcoxon rank sum $(p=0.10)$. Four patients had T-scores for depressive problems greater than or equal to 70 , the clinical cutoff, compared with one control.

As expected, RAVLT delayed free recall significantly differed between TLE patients and controls (Wilcoxon rank sum test $-p=0.008)$. No significant relationship was found between the number of antiepileptic drugs and the TLE patients' RAVLT delayed free recall (Spearman's rho, $\tilde{\rho}=0.07, \mathrm{~S}=756.23, p=0.780)$.

\section{Motion}

A histogram of the mean DVARS for all ECP participants was constructed to determine a mean DVARS cutoff value pertaining to excessive motion. Participants were included in this analysis if the pair of oppositely phase-encoded scans had a mean DVARS of $<60$. No significant difference in mean DVARS was found between patients and controls after averaging over phase encode $\left(t_{31}=-0.32, p=0.751\right)$.

\section{$D C M$ connections and AEDs}

No significant relationship between the spDCM connection strengths and the number of AEDs was found when investigated either dichotomously (monotherapy vs. polytherapy) or as a continuous variable ( $0-4$ medications) after corrections for multiple comparisons. Thus, the number of AEDs was not included in the PEB models.

\section{PEB models}

PEB model results will first be discussed for the model comparing TLE versus control participants, followed by the model that includes RAVLT delayed free recall index as a covariate. The final PEB models were obtained after an automatic search over nested models using model evidence. The number of parameters in each model was further reduced by only considering connections for which strong evidence (posterior probability $>0.95$ ) existed. The intercept demonstrates the common effective connectivity between the two groups, while the group variable encodes the differences in effective connectivity between TLE patients and controls. The phase encode variable was included in the model to account for potential variability attributable to the phase encode direction of the MR scan.

\section{Model 1-TLE versus control participants}

Figure 1 shows the final spDCM of the DMN for the group mean. All DMN regions had strong evidence for a self- 


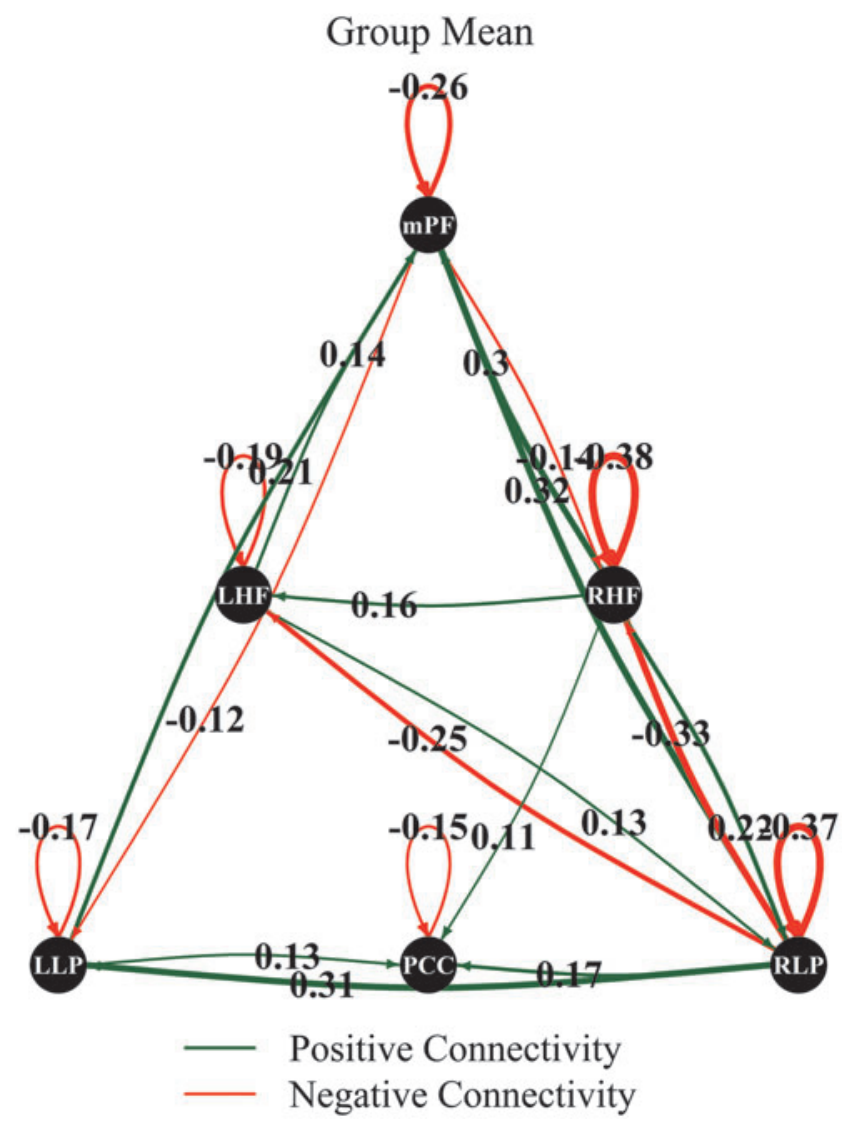

FIG. 1. A network view of the thresholded connections of the DMN with strong evidence for the common group mean from the without RAVLT PEB model. Connections are color-coded based on if the connection was inhibitory (red) or excitatory (green). The thickness of the line and size of the arrowheads are indicative of the strength of the connection, listed near the terminating region. PCC, posterior cingulate cortex; $\mathrm{mPF}$, medial prefrontal cortex; LLP, left lateral parietal cortex; RLP, right lateral parietal cortex; LHF, left hippocampal formation; RHF, right hippocampal formation; RAVLT, Rey Auditory Verbal Learning Test; PEB, parametric empirical Bayes; DMN, default mode network.

connection. All DMN self-connections in the group mean are less self-inhibiting compared with the prior mean due to their negative values. Strong evidence showed the following connections to be excitatory: LLPC to PCC, LLPC to MPFC, RLPC to PCC, RLPC to mPFC, RLPC to LLPC, LHF to mPFC, LHF to RLPC, RHF to PCC, RHF to mPFC, RHF to RLPC, and RHF to LHF. The following connections were found to be inhibitory: mPFC to LLPC, mPFC to RHF, RLPC to LHF, and RLPC to RHF.

Figure 2 shows the final spDCM of the DMN for the group difference. Self-connections did not differ between TLE patients and controls. Three connections lead to greater excitation in controls compared with patients: PCC to LHF, RLPC to RHF, and RHF to mPFC. The one connection leading to greater excitation in TLE patients compared with controls was LHF to PCC. Inhibition of LHF in the left TLE population may result from these connection differences.

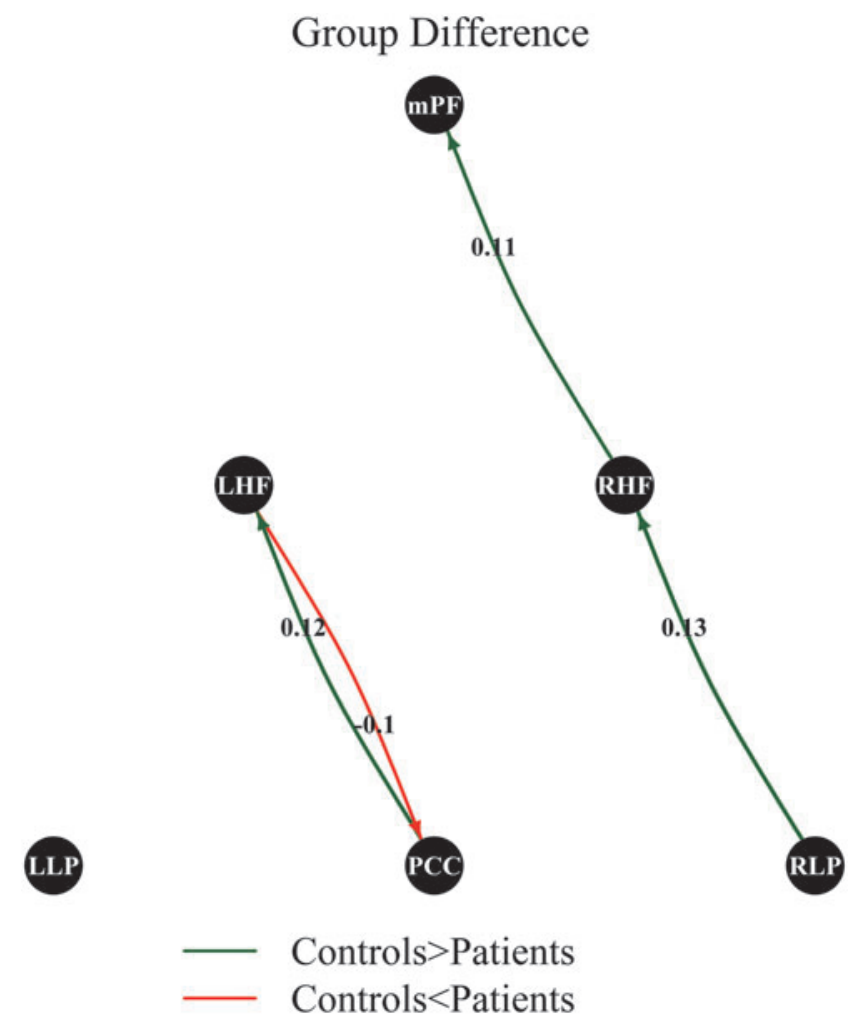

FIG. 2. A network view of the thresholded connections of the DMN with strong evidence (posterior probability $>0.95$ ) for a difference between left TLE patients and controls from the without RAVLT PEB model. Connections are colorcoded based on if the difference in connection strengths was larger in controls compared with patients (green) or larger in patients compared with controls (red). The thickness of the line and size of the arrowheads are indicative of the strength of the connection, listed near the terminating region. TLE, temporal lobe epilepsy.

\section{Model 2-TLE versus control participants with delayed verbal memory as covariate}

Strong evidence was found for all self-connections for the group common effective connectivity (Fig. 3). The following connections were found to be excitatory: PCC to mPFC, RLPC to PCC, RLPC to mPFC, LHF to mPFC, RHF to PCC, RHF to mPFC, RHF to LLPC, RHF to RLPC, and RHF to LHF. Inhibitory connections were found for the following: mPFC to LLPC, mPFC to RLPC, mPFC to RHF, RLPC to LHF, and RLPC to RHF.

Five connections differed between the two groups (Fig. 4). Four of them were in the previous model with the same group having a more excitatory connection: PCC to LHF, RLPC to RHF, LHF to PCC, and RHF to mPFC. Thus, these connection differences are not attributed to memory differences alone. PCC to mPFC was found to be more excitatory for patients compared with controls. PCC to mPFC was also found to be associated with an increase in the RAVLT delayed free recall (Fig. 5). This interplay of group difference and association with increasing RAVLT for connection strength from PCC to mPFC may be concomitant with the LHF inhibition noted in TLE patients. 


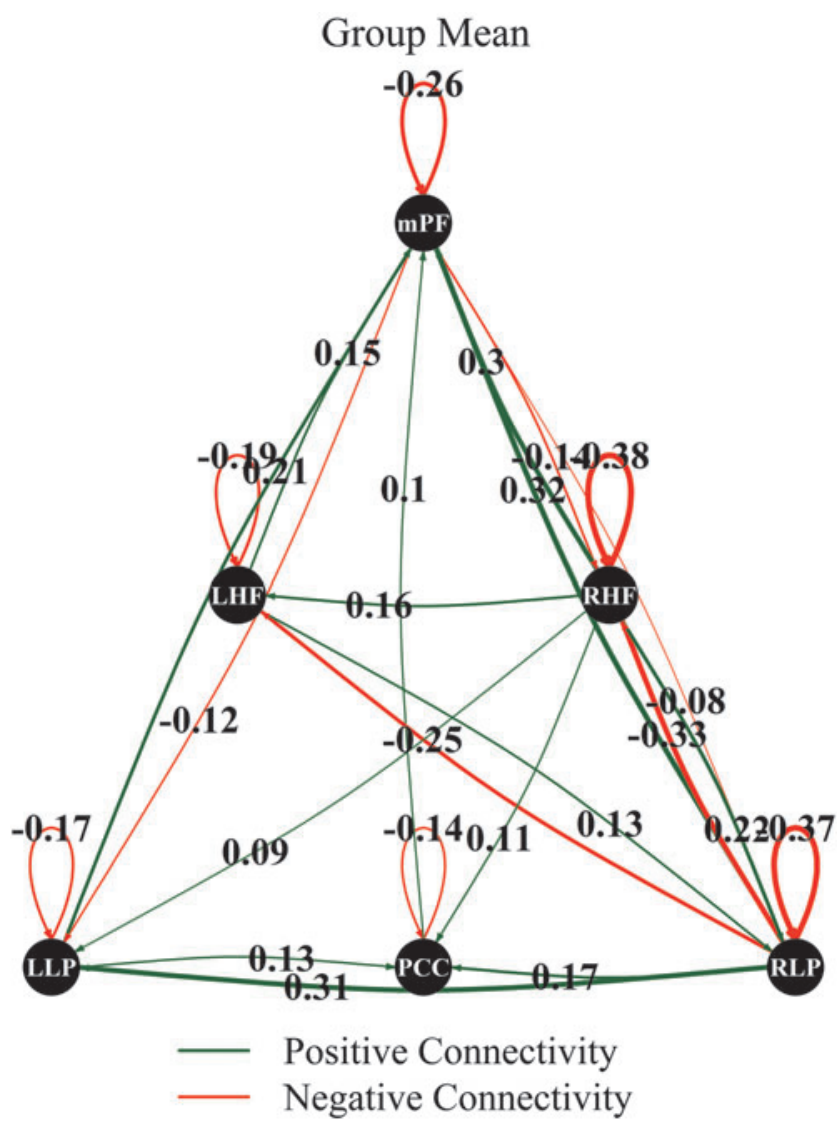

FIG. 3. A network view of the thresholded connections of the DMN with strong evidence (posterior probability $>0.95$ ) for the common group mean with RAVLT delayed free recall equal to the grand mean from the with RAVLT PEB model. Connections are color-coded based on if the connection was inhibitory (red) or excitatory (green). The thickness of the line and size of the arrowheads are indicative of the strength of the connection, listed near the terminating region.

\section{Discussion}

The DMN is implicated in episodic, autobiographical, and semantic memory processes (Binder et al., 2009; Buckner et al., 2008; Sestieri et al., 2011). TLE patients commonly exhibit multiple memory impairments affecting autobiographical (Herfurth et al., 2010; St-Laurent et al., 2009), spatial (Amlerova et al., 2013; Glikmann-Johnston et al., 2008), and semantic systems (Giovagnoli, 1999). Verbal episodic memory disruption is frequently associated with left TLE (Grammaldo et al., 2006; Loring et al., 2008) and significantly poorer delayed recall was observed here as hypothesized. Using spDCM, effective connectivity was compared between left TLE patients and controls using PEB models. Two second-level models were investigated to this end, one without and the other with RAVLT score as a covariate, the latter controlling for the potential impact of psychometric memory differences on group effective connectivity patterns. Incorporating RAVLT into the model investigates what, if any, group-level connectivity differences exist after accounting for individual differences in memory, a function of the DMN.

A core finding, derived from this investigation when comparing TLE patients to controls, was four connections (PCC

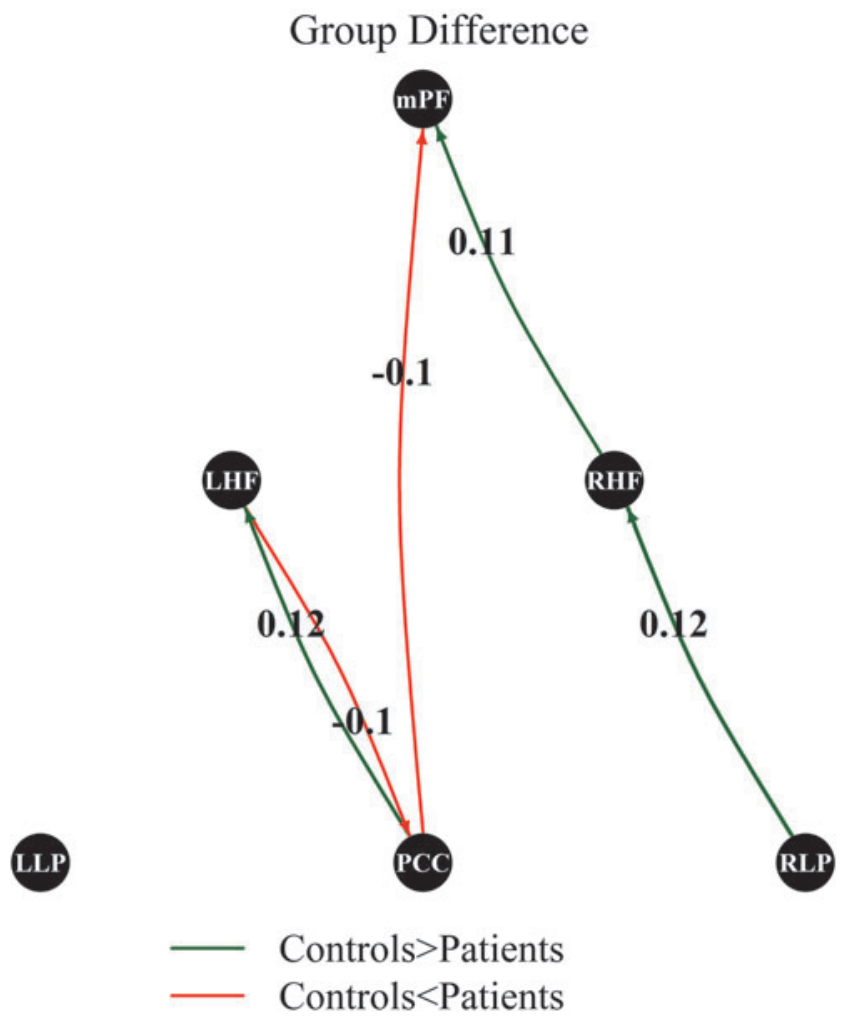

FIG. 4. A network view of the thresholded connections of the DMN with strong evidence (posterior probability $>0.95$ ) for a difference between patients and controls with RAVLT delayed free recall equal to the grand mean from the with RAVLT PEB model. Connections are color-coded based on if the difference in connection strengths was larger in controls compared with patients (green) or larger in patients compared with controls (red). The thickness of the line and size of the arrowheads are indicative of the strength of the connection, listed near the terminating region.

to $\mathrm{LHF}$, LHF to PCC, RLPC to RHF, and RHF to mPFC) that differed between groups in both models, potentially serving to inhibit LHF activation. A core association when including RAVLT delayed free recall was an excitatory connection (PCC to mPFC) concomitant with LHF inhibition in TLE patients after accounting for RAVLT delayed free recall. Below we discuss the findings from each of the models in greater detail.

\section{Model 1-TLE versus control participants}

Four connections differed between TLE patients and controls in the verbal memory performance independent model (Fig. 2). PCC to LHF was found to be excitatory in controls and inhibitory in TLE patients. LHF to PCC, conversely, was found to be excitatory in TLE patients and inhibitory in controls. The magnitude of the connection strength from PCC to LHF is larger than that of LHF to PCC. These strength differences and the loop created by these two connections are likely due to the left temporal lobe epileptogenic focus for the TLE patients. Inhibition of the LHF may also occur as a result of the other two connections that differ between groups. The connection from RLPC to RHF was found to be more excitatory in controls compared with TLE patients, and thus, more inhibitory in TLE patients compared with 


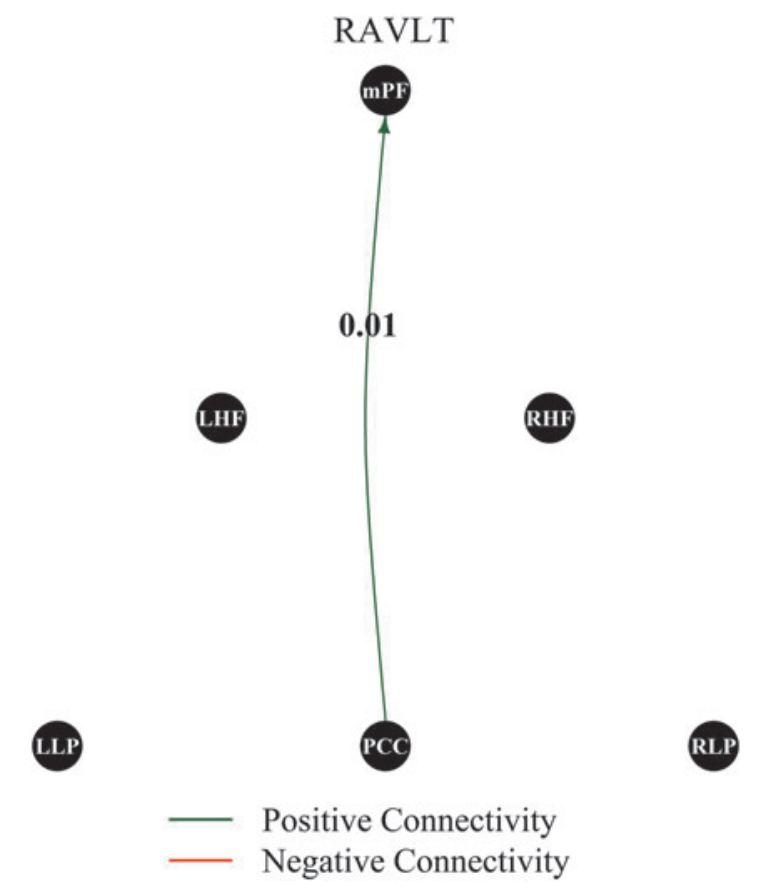

FIG. 5. A network view of the thresholded connections of the DMN with strong evidence (posterior probability $>0.95$ ) for a difference with each increase in RAVLT delayed free recall from the grand mean from the with RAVLT PEB model. Connections are color-coded based on if the connection was inhibitory (red) or excitatory (green). The thickness of the line and size of the arrowheads are indicative of the strength of the connection, listed near the terminating region.

controls. Neuronal activity in the RLPC of TLE patients would lead to inhibition of RHF, reducing the ability for RHF to excite LHF, a connection in the group mean network (Fig. 1). The final connection that differed between TLE patients and controls, RHF to mPFC, was more excitatory in controls. Together, with the connections from the group mean network (Fig. 1), RHF would then be inhibited from mPFC, which would reduce the amount of excitation of LHF from RHF. The LHF has an important role in verbal memory (Burgess et al., 2002). Thus, the observed LHF inhibition through various pathways may explain the often noted verbal memory differences in left TLE patients and controls or be a result of epileptogenic focus.

\section{Model 2-TLE versus control participants with verbal memory covariate}

The four connections that differed between the two groups, which were seen in the previous model, may again cause inhibition of LHF (Fig. 4). Although the LHF has an important role in verbal memory (Burgess et al., 2002), these connections leading to inhibition of LHF still exist after accounting for verbal memory differences. Thus, these four connectivity differences may stem from the epileptogenic focus in the left hemisphere, potentially attempting to mitigate seizure propagation.

In addition, increasing RAVLT delayed free recall was associated with an increase in excitation of mPFC from PCC (Fig. 5). This connection was found to be more excitatory in TLE patients and more inhibitory in controls (Fig. 4). This may allow mPFC to use additional autobiographical memories retrieved from PCC (Maddock et al., 2001) to aid performance and be concomitant with the increased inhibition of LHF found in TLE patients.

\section{Limitations}

One study limitation is the modest sample size of the left TLE group, but this also provided a homogenous sample unconfounded by varying laterality of seizure onset. Replication with a larger sample size will help to confirm and extend these findings. Further analyses are needed to determine if similar results would be obtained for right TLE subjects with nonverbal/visual memory as a covariate. Another issue for future research is whether these patterns differ based on the structures involved, that is, mesial versus neocortical TLE. In addition, the effective connectivity differences between TLE patients who respond to treatment versus those with refractory epilepsy would be valuable to explore, as well.

The current TLE patient sample was largely a nonsurgical sample in nature and therefore did not routinely undergo ictal monitoring. Therefore, the presence and/or number of possible comorbid nonepileptic seizures cannot be determined. However, all TLE patients were undoubtedly diagnosed and treated for TLE, given the inclusion criteria. For example, incidence of epileptiform discharges in a nonepileptic population is very low (Gregory et al., 1993). The current sample is likely a more representative sample of the TLE population as a whole, which could be viewed as reducing the limitations induced by lack of monitoring.

Currently, the noted group differences in effective connectivity cannot be ruled out as being affected in some way by AED use. All but one patient were currently using at least one AED. Some AEDs have been demonstrated to alter neuronal activation (Wandschneider et al., 2014). Further investigation is needed to determine the effects of presence versus absence of AED treatment on effective connectivity.

\section{Conclusion}

ECP aims to map connectome differences between TLE patients and healthy controls. To this end, spDCM was used to investigate differences in effective connectivity in the DMN between individuals with left TLE and healthy controls using ECP data. Two PEB models were formulated to investigate these differences; one that accounted for individual differences in memory performance using RAVLT delayed free recall and the other that did not.

All models demonstrated a cluster of four connections that differed in left TLE patients and controls, which may serve to inhibit LHF activation in patients. Thus, inhibition of the LHF in left TLE patients may be a result of the left temporal lobe epileptogenic focus. Greater excitation from PCC to mPFC was found to be concomitant with LHF inhibition in TLE patients and this connection was associated with greater excitation with increasing RAVLT delayed free recall.

\section{Acknowledgments}

The authors thank all the participants and their families. In addition, the authors thank Taylor McMillan for recruitment 
and participant acquisition, MRI technologists for their assistance in scanning, and other support staff. They also thank the reviewers for their helpful and insightful comments, assisting in clarifying and strengthening this work. Funding for healthy control subjects' data acquisition was provided, in part, by the Department of Radiology at the University of Wisconsin-Madison. This study was supported by grant number U01NS093650 from the National Health Institute (J.R.B. and M.E.M. Co-PIs).

\section{Author Disclosure Statement}

No competing financial interests exist.

\section{References}

Achenbach TM, Newhouse PA. 2004. Manual for the ASEBA Older Adult Forms and Profiles. Burlington, VT: University of Vermont, Research Center for Children, Youth, and Families.

Amlerova J, Laczo J, Vlcek K, Javurkova A, Andel R, Marusic P. 2013. Risk factors for spatial memory impairment in patients with temporal lobe epilepsy. Epilepsy Behav 26:57-60.

Binder JR, Desai RH, Graves WW, Conant LL. 2009. Where is the semantic system? A critical review and meta-analysis of 120 functional neuroimaging studies. Cereb Cortex 19:27672796.

Buckner RL, Andrews-Hanna JR, Schacter DL. 2008. The brain's default network: anatomy, function, and relevance to disease. Ann N Y Acad Sci 1124:1-38.

Burgess N, Maguire EA, Keefe JO. 2002. The human hippocampus and spatial and episodic memory. Neuron 35:625-641.

Cataldi M, Avoli M, De Villers-Sidani E. 2013. Resting state networks in temporal lobe epilepsy. Epilepsia 54:2048-2059.

Dale A, Fischl B, Sereno MI. 1999. Cortical surface-based analysis: I. Segmentation and surface reconstruction. Neuroimage 9:179-194.

David O, Guillemain I, Saillet S, Reyt S, Deransart C, Segebarth C, Depaulis, A. 2008. Identifying neural drivers with functional MRI: an electrophysiological validation. PLoS Biol 6:e315.

Fischl B, Dale AM. 2000. Measuring the thickness of the human cerebral cortex from magnetic resonance images. Proc Natl Acad Sci U S A 97:11050-11055.

Fischl B, Salat DH, Busa E, Albert M, Dieterich M, Haselgrove C, et al. 2002. Whole brain segmentation: automated labeling of neuroanatomical structures in the human brain. Neuron 33: 341-355.

Fisher RS. 2013. Temporal lobe epilepsy (TLE). www.epilepsy .com/learn/types-epilepsy-syndromes/temporal-lobe-epilepsyaka-tle Last accessed Dec. 12, 2017.

Friston KJ. 2011a. Dynamic causal modeling and Granger causality Comments on: the identification of interacting networks in the brain using fMRI: model selection, causality and deconvolution. Neuroimage 58:303-305.

Friston KJ. 2011b. Functional and effective connectivity: a review. Brain Connect 1:13-36.

Friston KJ, Bastos AM, Oswal A, van Wijk B, Richter C, Litvak V. 2014. Granger causality revisited. Neuroimage 101:796-808.

Friston KJ, Harrison L, Penny W. 2003. Dynamic causal modelling. Neuroimage 19:1273-1302.

Friston KJ, Kahan J, Biswal B, Razi A. 2014. A DCM for resting state fMRI. Neuroimage 94:396-407.

Friston KJ, Litvak V, Oswal A, Razi A, Stephan KE, Van Wijk BCM, et al. 2016. Bayesian model reduction and empirical Bayes for group (DCM) studies. Neuroimage 128:413-431.
Friston KJ, Mattout J, Trujillo-Barreto N, Ashburner J, Penny W. 2007. Variational free energy and the Laplace approximation. Neuroimage 34:220-234.

Friston KJ, Moran R, Seth AK. 2013. Analysing connectivity with Granger causality and dynamic causal modelling. Curr Opin Neurobiol 23:172-178.

Geffen G, Hoar KJ, O’Hanlon AP, Clark CR, Geffen LB. 1990. Performance measures of 16- to 86-year-old males and females on the auditory verbal learning test. Clin Neuropsychol $4: 45-63$.

Giovagnoli AR. 1999. Verbal semantic memory in temporal lobe epilepsy. Acta Neurol Scand 99:334-9.

Glasser MF, Sotiropoulos SN, Wilson JA, Coalson TS, Fischl B, Andersson JL, et al. 2013. The minimal preprocessing pipelines for the Human Connectome Project. Neuroimage 80:105-124.

Glikmann-Johnston Y, Saling MM, Chen J, Cooper KA, Beare RJ, Reutens DC. 2008. Structural and functional correlates of unilateral mesial temporal lobe spatial memory impairment. Brain 131:3006-3018.

Grammaldo LG, Giampa T, Quarato PP, Picardi A, Mascia A, Sparano A, et al. 2006. Lateralizing value of memory tests in drugresistant temporal lobe epilepsy. Eur J Neurol 13:371-376.

Gregory R, Oates T, Merry R. 1993. Electroencephalogram epileptiform abnormalities in candidates for aircrew training. Electroencephalogr Clin Neurophysiol 86:75-77.

Helmstaedter C, Witt J. 2012. Clinical neuropsychology in epilepsy: theoretical and practical issues. Handb Clin Neurol 107:437-59.

Herfurth K, Kasper B, Schwarz M, Stefan H, Pauli E. 2010. Autobiographical memory in temporal lobe epilepsy: role of hippocampal and temporal lateral structures. Epilepsy Behav 19:365-371.

Holmes MD, Dodrill CB, Wilensky AJ, Ojemann LM, Ojemann GA. 1996. Unilateral focal preponderance of interictal epileptiform discharges as a predictor of seizure origin. Arch Neurol 53:228-232.

Jenkinson M, Beckmann CF, Behrens TE, Woolrich MW, Smith SM. 2012. FSL. Neuroimage 62:782-90.

Lin JJ, Mula M, Hermann BP. 2012. Uncovering the neurobehavioral comorbidities of epilepsy over the lifespan. Lancet 380:1180-1192.

Loring DW, Strauss E, Hermann BP, Barr WB, Perrine K, Trenerry MR, et al. 2008. Differential neuropsychological test sensitivity to left temporal lobe epilepsy. J Int Neuropsychol Soc 14:394-400.

Luo C, Yang F, Deng J, Zhang Y, Hou C, Huang Y, et al. 2016. Altered functional and effective connectivity in anticorrelated intrinsic networks in children with benign childhood epilepsy with centrotemporal spikes. Medicine (Baltimore) 95: e3831.

Maddock RJ, Garrett AS, Buonocore MH. 2001. Remembering familiar people: the posterior cingulate cortex and autobiographical memory retrieval. Neuroscience 104:667-676.

McCormick C, Quraan M, Cohn M, Valiante TA, McAndrews MP. 2013. Default mode network connectivity indicates episodic memory capacity in mesial temporal lobe epilepsy. Epilepsia 54:809-818.

Park HJ, Pae C, Friston KJ, Jang C, Razi A, Zeidman P, et al. 2017. Hierarchical dynamic causal modeling of restingstate fMRI reveals longitudinal changes in effective connectivity in the motor system after thalamotomy for essential tremor. Front Neurol 8:1-9.

Power JD, Barnes KA, Snyder AZ, Schlaggar BL, Petersen SE. 2012. Spurious but systematic correlations in functional 
connectivity MRI networks arise from subject motion. Neuroimage 59:2142-2154.

R Core Team. 2017. R: A Language and Environment for Statistical Computing. Vienna, Austria: R Foundation for Statistial Computing. www.r-project.org/ Last accessed June 24, 2017.

Rey AL. 1941. Psychological examination of traumatic encephalopathy. Arch Psychol 28:286-340.

Saling MM. 2009. Verbal memory in mesial temporal lobe epilepsy: beyond material specificity. Brain 132(Pt 3):570-592.

Schmidt M. 2017. (RAVLT) Rey auditory verbal learning test. www.wpspublish.com/store/p/2933/ravlt-rey-auditory-verballearning-test Last accessed February 15, 2018.

Sestieri C, Corbetta M, Romani GL, Shulman GL. 2011. Episodic memory retrieval, parietal cortex, and the default mode network: functional and topographic analyses. J Neurosci 31:4407-4420.

Sirven JI, Shafer PO. 2014. What is epilepsy? www.epilepsy.com/ learn/about-epilepsy-basics/what-epilepsy Last accessed Dec. 12, 2017.

St-Laurent M, Moscovitch M, Levine B, McAndrews MP. 2009. Determinants of autobiographical memory in patients with unilateral temporal lobe epilepsy or excisions. Neuropsychologia 47:2211-2221.

Stephan KE, Weiskopf N, Drysdale PM, Robinson PA, Friston KJ. 2007. Comparing hemodynamic models with DCM. Neuroimage 38:387-401.

The FIL Methods Group. 2016. Statistical Parametric Mapping 12. London, United Kingdom: FIL. www.fil.ion.ucl.ac.uk/ spm/ Last accessed October 1, 2017.

Theodore WH, Wiggs EA, Martinez AR, Dustin IH, Khan OI, Appel S, et al. 2012. Serotonin 1A receptors, depression, and memory in temporal lobe epilepsy. Epilepsia 53:129-133.

Van Dijk KRA, Hedden T, Venkataraman A, Evans KC, Lazar SW, Buckner RL. 2010. Intrinsic functional connectivity as a tool for human connectomics: theory, properties, and optimization. J Neurophysiol 103:297-321.
Voets NL, Beckmann CF, Cole DM, Hong S, Bernasconi A, Bernasconi N. 2012. Structural substrates for resting network disruption in temporal lobe epilepsy. Brain 135:2350-2357.

Vollmar C, Stredl I, Heinig M, Noachtar S, Remi J. 2018. Unilateral temporal interictal epileptiform discharges correctly predict the epileptogenic zone in lesional temporal lobe epilepsy. Epilepsia 59:1577-1582.

Wandschneider B, Stretton J, Sidhu M, Centeno M, Kozak LR, Symms M, et al. 2014. Levetiracetam reduces abnormal network activations in temporal lobe epilepsy. Neurology 83: 1508-1512.

Wei H, An J, Shen H, Zeng LL, Qiu S, Hu D. 2016. Altered effective connectivity among core neurocognitive networks in idiopathic generalized epilepsy: an fMRI evidence. Front Hum Neurosci 10:1-15.

Williamson PD, French JA, Thadani VM, Kim JH, Novelly RA, Spencer SS, et al. 1993. Characteristics of medial temporal lobe epilepsy: II. Interictal and ictal scalp electroencephalography, neuropsychological testing, neuroimaging, surgical results, and pathology. Ann Neurol 34:781-787.

Yaffe RB, Borger P, Megevand P, Groppe DM, Kramer MA, Chu CJ, et al. 2015. Physiology of functional and effective networks in epilepsy. Clin Neurophysiol 126:227-236.

Zhang Z, Lu G, Zhong Y, Tan Q, Liao W, Wang Z, et al. 2010. Altered spontaneous neuronal activity of the default-mode network in mesial temporal lobe epilepsy. Brain Res 1323: $152-160$.

Address correspondence to: Mary E. Meyerand Department of Medical Physics University of Wisconsin-Madison 1111 Highland Avenue, Rm 1005 Madison, WI 53705

E-mail: memeyerand@wisc.edu 